Ageing and Society 21, 200 I, 319-332. (C) 200 I Cambridge University Press

DOI : гогі 7/Sor44686 roor oo825x Printed in the United Kingdom

\title{
Life and death in English nursing homes: sequestration or transition?
}

\author{
KATHERINE FROGGATT*
}

\begin{abstract}
Nursing homes as care institutions seek to offer a home where people can live until their death. A potential conflict, therefore, exists as nursing homes are both a place where life is lived and where death is regularly encountered. It has been proposed that within residential care homes for older people, dying individuals are separated from living people. Drawing on fieldwork undertaken in four English nursing homes the management of the dying process and the relationship between life and death is explored. There is much uncertainty inherent in the boundary between life and death for many residents in nursing homes. The relationship between life and death for these residents is less about the sequestration of dying people from living people, but rather the creation of transitional states between these two polarities.
\end{abstract}

$\boldsymbol{K} \boldsymbol{E} \boldsymbol{Y} \boldsymbol{W O R} \boldsymbol{D} \boldsymbol{S}-$ Nursing homes, sequestration, death and dying, social death.

\section{Introduction}

It has been proposed that within Western society dying and ageing people are separated, geographically and socially, from younger 'living' people (Mulkay and Ernst r99I). This setting apart of dying people has also been observed within institutions that care for older people (Hockey r 990: 98-гог; Mulkay and Ernst i99г: г83). The nature of the relationship between living and dying residents in nursing homes and the management of this transition is explored within this paper drawing upon fieldwork undertaken in four English nursing homes.

\section{Sequestration and society}

Giddens' work concerning the nature of modern society and the individual proposes that we currently live in a time of great change and

* Macmillan Practice Development Unit, The Institute of Cancer Research, London. 
movement at global and individual levels (Giddens r99i). As a consequence, the reflexive reordering of biographical narratives shapes self-identity, and there is an increasing identification of the self with the body (Mellor and Shilling r 993). Events that may interfere with the regularity of daily life are controlled, primarily through the setting apart of these experiences so they do not impinge upon the rest of society. Sickness and death, madness and criminality are all experiences that are concealed and set apart from the routines of ordinary life (Giddens i 991 : I56).

The process of individualisation that characterises modernity, and the lack of the religious structures and practices which formerly shaped society, means that people cannot easily incorporate the inevitability of death. As a consequence it has become a relatively hidden experience (Mellor and Shilling r 993) and the boundaries surrounding living and dying people strengthened. This is manifested particularly in the creation of spaces where the bodily evidence of sickness and death are set apart, that is, in hospitals (Giddens I99 I Mellor and Shilling I993), hospices (Mellor I993; Mellor and Shilling I993), nursing homes and residential care homes (Hockey i 990; Mulkay and Ernst I 99 I).

Sequestration is the term given to this process of setting apart or separating out. With respect to death and dying the term has been used in two ways. First, at a societal level, sequestration has been used as a concept to describe the way in which contemporary 'modern' society has set death apart from the ordinary lives of individual people. This may be facilitated through the use of organisations (Giddens ig9 I Mellor I993; Mellor and Shilling i993) or through the more general characteristics of the systems of modernity (Giddens i99I). Secondly, the model of sequestration has been used to explain the separation of defined groups of people within an institution. Dying older people in residential care homes are kept apart from those residents who are perceived to be living (Hockey r 990) and in hospices patients with 'unbounded' bodies are separated from other patients whose bodies remain 'bounded' (Lawton I 998).

Another manifestation of the sequestration of death and dying within society can be seen in the presence of social death, which represents the setting apart of people from a social perspective. Social death has been defined as the cessation of the individual as an active agent in other people's lives (Glaser and Strauss i 965; Sudnow i967; Glaser and Strauss i 968; Mulkay and Ernst i99 I Hertz i960). Social death is linked to, but not predicated upon, bodily death, and social death increasingly occurs in Western society before the biological death event. 
Social death is particularly pertinent when considering the move from life to death amongst older people. The emphasis on the future, present within high modernity, is incorporated by younger people, not older people whose future is more obviously limited (Mellor and Shilling i 993). As a consequence, the subject of ageing is avoided, and older people are socially marginalised (Hockey and James i 993). There are several ways in which this marginalisation is manifested. These include the act of retirement which signifies a leaving of social roles occupied in society, the physical separation or institutionalisation of older people which leads to a distancing from social networks, and the presence of anticipatory grief in relatives (Mulkay and Ernst I99 I). Sweeting and Gilhooly go so far as to propose that three groups of people have been identified as being socially dead within society (Sweeting and Gilhooly I99I-I992; Sweeting and Gilhooly 1997). They define these groups as very old people, those individuals at the end stage of a long term chronic illness, and people who are perceived to have lost their personhood, akin to Goffman's notion of the 'nonperson' (Goffman i 959).

Society, therefore, copes with the reality of death, and ageing as a precursor to death, by setting apart the people who bear visible evidence of the processes of dying. A variety of institutions provide space for this sequestration, for example hospitals and hospices, and this paper focuses on the nature of this separation within one type of care institution for older people - nursing homes.

\section{Methods}

The data considered in this paper are drawn from an evaluation of the 'Palliative Care Education Pilot Project in Nursing Homes' (Froggatt $2000 a, 2000 b$ ), funded by Macmillan Cancer Relief and The Wolfson Foundation in partnership with the Registered Nursing Home Association. In this evaluation, four case study nursing homes were purposively sampled from the 54 nursing homes that had participated in the educational project. Only the 38 nursing homes where both registered nurses and health care assistants had undertaken the educational courses were included (the remaining i 6 nursing homes had only sent registered nurses to the educational courses). In order to ensure the representativeness of these sites, the four case study nursing homes were selected to represent the following range of factors:

- nursing homes from four different health authorities;

- nursing homes that were independently owned by an individual, and nursing homes that were owned by a commercial group; 
T A в LE I. Case study nursing homes

\begin{tabular}{lcccc}
\hline Nursing home $^{1}$ & Daisey Lodge & Parkleigh & Braelands & Fairgreen \\
\hline $\begin{array}{l}\text { Average number of beds } \\
\begin{array}{c}\text { Ancillary staff on the } \\
\text { educational course }\end{array}\end{array}$ & 35 & 30 & 65 & 30 \\
$\begin{array}{l}\text { Registered nurses doing } \\
\text { assignment }\end{array}$ & Yes & Yes & No & No \\
$\begin{array}{l}\text { Health care assistants doing } \\
\text { assignment }\end{array}$ & Yes & Yes & No & No \\
$\begin{array}{l}\text { Ownership } \\
\text { Palliative care beds }\end{array}$ & $\begin{array}{c}\text { Single home } \\
\text { owner }\end{array}$ & $\begin{array}{c}\text { Single home } \\
\text { owner }\end{array}$ & Group home & $\begin{array}{c}\text { Single home } \\
\text { owner }\end{array}$ \\
\hline
\end{tabular}

1 The names of the case study nursing homes have been changed to preserve their anonymity.

- nursing homes with and without allocated palliative care beds;

- nursing homes where course members undertook assignments, and nursing homes where staff chose not to undertake assignments.

Details of the four nursing homes identified are presented in Table I. Upon being approached, all agreed to participate in the study. Within each of the four nursing homes the researcher, who worked as a care assistant, carried out a three-week period of participant observation. Although focusing primarily upon the impact of an educational programme upon the practice of nursing and care staff, the approaches to care and the nature of death and dying within the nursing home context were also observed. A total of 4I I hours of observational fieldwork was undertaken during these four visits. A variety of shifts were worked including one weekend and one night in each nursing home to give a broad overview of nursing home work.

During this time, 43 interviews were carried out with staff at all levels, including home owners, registered nurses, health care assistants and ancillary workers. The interviews focused on the impact of the educational courses undertaken, the nature of the care work and the place of death and dying within the nursing home. All interviews were transcribed and, along with the observational fieldnotes, were analysed using a computer-based qualitative data analysis package (NUD*IST), which facilitated the coding of the data for recurrent themes and issues.

\section{Sequestration or transition?}

It has been proposed that, within some institutions caring for older people such as residential care homes, sequestration exists to separate 
those close to death from those whose death is less imminent (Hockey I990). Hockey identified that the residents in the residential home she studied were classified as either 'fit' or 'frail' and that this had implications for the spatial locations, social roles occupied and care received by these residents (Hockey i 990). This division between the 'fit' and the 'frail' represented a separation between the living and the dying within residential homes. Within nursing homes, however, the boundary between life and death is not so clearly defined, reflecting the functions that nursing homes as institutions fulfil and the dying trajectories experienced by many residents in this setting.

At a societal level, nursing homes are imputed with the task of containing the visible manifestations of ageing by society (Hockey i 990) and offer a form of social control over people who are no longer independent (Peace et al. I 997). More pragmatically, a tension was created for the case study nursing homes in that they cared for living and dying residents whilst being organisationally positioned in the private sector of English healthcare.

The four case study nursing homes were businesses, situated outside the publicly-financed National Health Service. They were owned variously by an individual, a group of individuals, and as part of a large commercial company. Through the provision of care for older people, the businesses aimed to make a profit for shareholders or owners. These nursing homes, in order to be successful, had to retain a high level of bed occupancy and offered both a home for older people as they lived there, and a place where nursing care could be provided when needed, or until the death of a resident.

Being a home was an important part of the ideology of the case study nursing homes. An emphasis on life and living permeated the culture of these four nursing homes. This manifested itself in a variety of ways, including their promotional literature; for example: 'We're opening up new lives for the elderly ... our residents too can look forward to a new lease of life' (Braelands Nursing Home promotional literature).

The reality of this new future was a life that was centred on the present, drawing on the strands of the past. This emphasis did not openly acknowledge the limited duration of the future and the inevitability that the majority of residents would die in the care of the nursing home. Interestingly, unlike the residential care home studied by Hockey (I990), these nursing homes also had an explicit commitment to nursing care, as described by this registered nurse:

The aim of the home is summed up in our mission statement, that we try to offer ... high quality nursing care in a homely type of atmosphere. Trying to 
treat people as if they are in their own homes, and try and remember that. But balancing it with the hospital-type nursing care environment. And that's often hard to do. (Parkleigh Nursing Home, registered nurse 2, course member)

The nursing homes held in balance being both a home and a place of nursing care. This can be seen in the terms used to describe the people who lived in the nursing homes. In three of them, they were called residents, whereas staff in the fourth called them patients. One of the nursing homes that used the term residents, however, produced literature for the care assistants that described the residents as patients, so capturing the ambiguity in the choice of language. In offering nursing care, nursing homes were following a health care model, whereby people were patients. Given the constraints on funding within the United Kingdom at the time of the study, nursing homes were seeking to identify their expertise as distinct from residential care homes. Making explicit the place of the health care model within their culture by, for example, calling the people who lived in the nursing home patients, was one way of achieving this.

The nature of the dying experienced by residents in nursing homes also shaped the life-death boundary. The way people died varied greatly, reflecting a range of ages and physical and mental conditions that residents lived with. In the four nursing homes visited, the ages of residents varied from 57 years to I I years. Unlike people with cancer where, until recently, the majority of people have experienced a relatively predictable dying trajectory, the dying processes of nursing home residents were generally longer-term and less certain.

In order to set dying people apart from living people, knowledge is required that allows an individual to be defined as dying. What has emerged from work that considers death amongst older people, is that the 'when' of death is much less clear than amongst younger people (Blackburn I989; Seale r989; Seale i 991). It has been recognised that situations of ageing and dying where the 'when' of death is unknown or unpredictable, are likely to be problematic for the dying individuals and those people caring for them (Field i 998). For elderly or confused people, or individuals living with a chronic disease, there exists uncertainty about a prognosis, and the absence of a diagnosis that defines them as being terminal. As McCue ( I 995) notes, a proportion of very elderly people die from 'natural' causes that cannot be attributed to a specific disease or condition, so creating situations where a prognosis is lacking. These people may therefore be denied access to the dying role, as defined by Glaser and Strauss ( 1965 , I 968 ), be it a modern dying role (Field i 996, I998) or a 'heroic' death (Seale i 995).

Death amongst older people has been considered from both a 
symptom management perspective (Wilson et al. I987; Mogielnicki et al. i 990), as well as from that of its physical, psychological, social and temporal dimensions (Blackburn i 989; Seale i 99 I Severs and Wilkins I99I). Sidell et al. (I997) retrospectively classified residential and nursing home resident deaths in four ways: sudden death, death following a terminal illness, death following an acute episode, and death as a process of general deterioration. The type of death which is least well understood, but which is significant in nursing homes, is the process of general deterioration. Although defined as a dying process in its own right, this may also be the stage that precedes an acute episode or sudden death. The process of general deterioration is a long-term one occurring over months and years.

It was described by staff in the nursing homes in a variety of ways. Residents were 'going downhill', people 'lingered' or were described as 'fading' or 'failing'. The following example, from one of the homes, illustrates this slow process of deterioration.

Mr Black, a 9 I-year-old gentleman, had been resident in Fairgreen Nursing Home for two-and-a-half years before his death. He had suffered a stroke before being admitted to the nursing home, and was also confused at times. As a consequence, he wandered around the nursing home from his room on the basement floor up to the ground floor lounge. The care staff described Mr Black as having 'rapidly gone down fast' over a period of three months. Having initially lost his voice, he then began to have difficulty in swallowing and eating and was losing weight. Mr Black's condition changed both physically and behaviourally, as noted by the staff I interviewed:

He just started going down. He was, always under your feet wandering up and down stairs and then, he was just always behind you and then all of a sudden he's just started going down. Nothing specific, just looking haggard, old. His colour, very, very breathless and then more fidgety than normal and then, from that, not even coming upstairs which isn't him. (Fairgreen Nursing Home, registered nurse 4)

The process of general deterioration described above was marked by changes in the resident's physical, mental or social health and subsequent dependency. The slow physical deterioration that represented the ageing and dying of many residents was not dramatic, but contained an element of uncertainty regarding their final resolution through death.

Staff in these nursing homes could not always predict when a resident would die as residents moved between the different life-death trajectories. There were instances where residents 'bounced back' from 
states of near death, as well as situations where they appeared to linger on before they finally died (Froggatt 2000b). An example of the 'bouncing back' phenomenon was Mrs Jones, a 72-year-old lady, who had been resident in Braelands Nursing Home for over three years. She had a chronic lung problem and arthritis, and since being admitted had suffered a minor stroke. Widowed with children, she had a male friend who visited her daily and often took her out for the day. One morning when I went in to work I heard that Mrs Jones was poorly, she had been ill overnight. She had been diagnosed as having had another stroke, the duty doctor having been called in to see her at 3 am that morning. Mrs Jones' male friend and son had been called in and were sitting with her during the night.

During that day, the door of her room was kept open and her friend and son could be seen sitting around the bed. As I left the nursing home that day, there was a sense of waiting for this death, particularly on the upstairs floor where her room was located. The next morning as I entered the dining room to collect breakfast for a resident, I was surprised to see Mrs Jones being pushed into the dining room in a wheelchair by her son. Again at lunchtime, things seemed to be back to normal, Mrs Jones occupied her usual place in the dining room, with her friend sitting next to her. The talk in the staff room that morning reminded everyone that this was not the first time Mrs Jones had done this. About nine months earlier she had become very ill, and had even had the Last Rites, before she had made an equally quick recovery.

This was an example of a resident who, seemingly stable, suddenly had an acute event, a stroke, which meant she was expected to die. The dynamics changed in readiness for a death. The wait began with family and friends present, and regular nursing care provided and overseen by the qualified nurse. However, within 24 hours Mrs Jones was reintegrated back into the life of the nursing home.

It was in this climate of uncertainty and, often prolonged, deterioration that many nursing home residents died. The care staff who worked in such institutions, both qualified nurses and health care assistants, had to make sense of this dying, whilst also caring for residents who were comparatively well and living their lives within their 'home'. For people whose condition deteriorated over many months and years, this lack of knowledge about when their death would occur meant that a clear distinction between when someone was living or dying was not possible. In such instances the transitional state (the living-dying interval) between life and death was as important as the absolute life and death categories themselves (Pattison i 977; Engle I998). 
'Being poorly'

An important category that was used by staff to manage the uncertainty was a transitional state called 'being poorly'. This was a definitional category that allowed staff to set apart residents who were ill, and also a category that conveyed meaning within the nursing home regarding the changes in a resident's condition (Hockey i 99o; Miskella and Avis I 998; Froggatt i 999). The term was used by staff, particularly during the handover of information from one shift to the next or when talking with relatives about a person's condition. Talking about a resident as 'poorly' sent signals to other members of staff and relatives that the condition had changed and that the person might die, whilst also acknowledging that this was not always the case.

'Being poorly' was a term that I heard being used by staff, residents and relatives to describe particular individuals. The primary attribute of being poorly was that the person, so defined, was perceived by others to be on a downward decline with respect to their state of health. The causes of being poorly were attributed either to an acute event, for example, staff talked about residents with a bowel obstruction or following a stroke as being poorly although, as in Mrs Jones' case, this state was not necessarily permanent. Sometimes there was no specific reason why someone was defined as being poorly and this state was attributed to a gradual, general decline, the slow process of deterioration described earlier.

The increased dependency of the resident whilst in this state would mean that the care staff would assume responsibility for more of the physical care of the residents which these individuals would formerly have undertaken for themselves. As a consequence, the individual concerned occupied a different space from other residents. He or she was more likely to be in their bedroom, and less involved in the activities that occurred in the public spaces. The following quote from a health care assistant in one of the case study nursing homes describes the separation of one such resident into his bedroom:

I think he's gone down a hell of a lot, an awful lot. ... Well say three weeks ago he was moving around in his wheelchair and eating and drinking on his own and he's gone to, in bed, not moving, and not eating and drinking. In the space of three weeks at the very most. (Braelands Nursing Home, health care assistant 2)

Within this transitional state, separation occurred which varied from day to day, even within a day, reflecting both the individual resident's condition and choices, and the different perceptions held by staff of the, 
primarily physical, state of residents. The occupation of different spaces and social roles whilst categorised as 'being poorly' indicated that there were some sequestration practices present within these institutions that mirrored the sequestration of death and ageing within wider society.

\section{Sequestration practices in nursing homes}

Staff set apart not only residents who were perceived to be poorly, as already described, but also residents who were perceived to have experienced a loss of personhood, or demonstrated signs of unbounded bodies. A loss of personhood was identified earlier as one of the causes of social death in contemporary society (Sweeting and Gilhooly I99I-I992). An individual resident could experience a loss of personhood either when their ability to communicate was impaired or when they possessed an unbounded body. Where an individual was unable to communicate rationally and clearly to staff, then decisions would be made on their behalf. This could occur where the person was confused, or because of a physical condition which limited their ability to communicate clearly such as a stroke.

Consequently, the residents who were able to communicate clearly were more able to make choices about their occupation of physical space in the nursing home, and the social roles they occupied. Those residents who, for whatever reason, were unable to communicate were not able to exert this choice. During the fieldwork, for example, I observed in all four nursing homes that, when residents were moved in readiness for mealtimes or prepared for bed, it was often those residents who were confused who would be moved or helped first. As one health care assistant commented about choosing particular residents to get ready for bed at $5 \mathrm{pm}$, 'they are confused and know no better'.

The individual with an unbounded body could also be perceived by others, or even by themselves, as no longer fully human. Lawton ( I 998, 2000) has suggested that, within contemporary hospices, it is people with cancer who were dying with unbounded bodies (fistulae, fungating tumours, lymphoedema) who were sequestered away from mainstream society and within the hospice. People who have such unbounded bodies experience a loss of self, and therefore encounter a social death before their bodily demise. For older people the deterioration of their body, physically and mentally, was also a significant reason for separation into institutional care. 
It is recognised that the nature of the unbounded body in the case study nursing homes was not as extreme as in Lawton's examples (Lawton I998). Incontinence was probably the most obvious way in which older people's bodies were unbounded. Attaining continence is seen as a developmental milestone amongst children, a sign of increasing maturity, whereas incontinence in older people is understood to be a marker of deterioration (Mitteness and Barker i 995). Other ways in which older people's bodies were increasingly unbounded were pressure sores, unhealed wounds, chest infections and the subsequent expectoration of infected sputum.

In general, people with unbounded bodies would only be set apart from other residents when the evidence of unboundedness could not be ignored. Where possible, boundaries would be created to contain the presence of unwanted bodily fluids. In cases of urinary continence, pads, or catheters if pads were not sufficient, would be used to create a boundary against the unboundedness of the urine. In the case of unhealed, weeping wounds dressings were applied. When these boundaries were breached people present in the public areas would be taken aside, back to their bedroom or to a bathroom, in order to reinstate the boundary. The signs of the body's breaching would be taken away with a change into clean and dry clothes or the application of new wound dressings.

Separation of residents occurred spatially and through their adoption of different social roles. The spatial separation of residents was seen most obviously in the four case study nursing homes, in the use of the public and private spaces. One indication as to whether residents were set apart from the wider community was the extent to which particular residents occupied the public areas of the nursing homes as opposed to spending time in their own rooms.

Further evidence of sequestration could be seen in the activities that occurred in the public spaces of the nursing homes. In the public lounges it was possible to observe a clear demarcation between the more able and mentally alert residents and those who were confused and less alert. In one nursing home this separation was most marked, with one bay of the lounge being occupied by the more able people and the other bay being occupied by those who were not engaging in any activity. This type of separation did in part reflect the physical layout of the nursing homes and the facilities they offered. If there was only one lounge then the separation occurred within this room. If there was more than one lounge, then the residents I observed were usually separated into the two rooms according to their ability socially to interact. 
The social roles occupied by residents were closely related to the spatial separation just described. As a consequence of the increasing dependency and need for nursing care that many residents demonstrated towards the end of their lives, individuals whose condition was deteriorating, or who were perceived by staff to be confused, would take on a different social role within the nursing home. This could be seen mainly in a resident's decreasing presence in the public areas of a nursing home - the dining room or lounge-or participation in recreational activities, and in a resident's increasing presence in his or her bedroom :

Alison hasn't been down to the lounge for some time. And I could, I could harness her. She was fairly flighty, and would come in and fly out, but I could harness her on occasions, and she would join in. But she's not coming down any more, she's not well enough. (Parkleigh Nursing Home, recreational coordinator)

Recreational activities usually took place within the lounge or another public room, and the level of participation would depend upon personal choice for those people able to decide. For those residents who could not communicate their needs, the location they found themselves in within the lounge would determine their level of participation. Those residents sitting close to the activity would be able to take part more easily, whereas those who were seated on the edges of the room, would be passively present rather than actively involved.

Individuals residing within nursing homes could and did choose to set themselves apart from the wider nursing home community. The degree to which they set themselves apart varied and was one indication of the social roles adopted by particular residents. Some spent their day in the public areas, others would only leave their bedroom for meals in the dining room, whereas others again would remain in their rooms all the time. In the following example, a registered nurse describes one resident who preferred to remain in her room all day, even at meal times.

But, for some, they don't want anything. They just want to be comfortable, like Nancy for example. She may just want to sit in a room, watch a television, have her visitors, do her knitting and talk to you when you go in, if you haven't noticed. That's her life, she's happy - she was doing that at home. (Daisey Lodge Nursing Home, registered nurse 3)

Residents who remained in their bedrooms might not be engaged with social roles within the nursing home, but could be focused on life without the nursing home, through visiting, letters or telephone contact with family and friends. 


\section{Conclusions}

The relationship between life and death in nursing homes is problematic. An institution provides well-bounded space to contain evidence of ageing and dying amongst older people, thereby sequestering people from wider society. Within the institution, however, the boundary is less clear. Living and dying occur in the same space, and staff members are required to care for residents, whatever their position on this continuum.

In using the concept of sequestration to explore the relationship between life and death, there was evidence in these nursing homes of the separation of residents both spatially and socially. Depending upon their degree of dependency and their underlying physical or mental condition, different spatial places were occupied and roles adopted. The uncertainty inherent in the passage from life to death for many older people who reside in nursing homes shaped the nature of the separation. Sequestration in these nursing homes was marked less by a clear separation of living residents from those who were dying than by the creation of transitional states between the two polarities that could be occupied by residents temporarily or permanently, often for many weeks and months.

\section{Acknowledgements}

I would like to thank the editor and two referees for their helpful comments.

\section{References}

Blackburn, A. M. ig89. Problems of terminal care in elderly patients. Palliative Medicine, 3, 203-6.

Engle, V. F. I 998. Care of the living, care of the dying: reconceptualising nursing home care. Fournal of the American Geriatric Society, 46, i I 72-4.

Field, D. i 996. Awareness and the modern role of dying. Mortality, I, 255-65.

Field, D. i 998. Special not different: General Practitioners' accounts of their care of dying people. Social Science and Medicine, 46, 9, I I I I-20.

Froggatt, K. A. I999. Evaluation of the palliative care education pilot project in nursing homes. Report for Macmillan Cancer Relief. Macmillan Cancer Relief, London.

Froggatt, K. $2000 a$. Evaluating a palliative care pilot project in nursing homes. International Fournal of Palliative Nursing, 6, 3, I $40-6$.

Froggatt, K. $2000 b$. Palliative care education in nursing homes. Abridged Report Produced for Macmillan Cancer Relief. Macmillan Cancer Relief, London.

Giddens, A. г991. Modernity and Self-Identity. Polity Press, Cambridge.

Glaser, B. G. and Strauss, A. L. I965. Awareness of Dying. Aldine, New York.

Glaser, B. G. and Strauss, A. L. I 968. Time for Dying. Aldine, New York.

Goffman, E. I 959. The Presentation of Self in Everyday Life. Penguin, Harmondsworth.

Hertz, R. I960(I907). Death and the Right Hand. The University Press, Aberdeen. 
Hockey, J. i99o. Experiences of Death. An Anthropological Account. Edinburgh University Press, Edinburgh.

Hockey, J. and James, A. 1993. Growing Up and Growing Old. Ageing and Dependency in the Life Course. Sage, London.

Lawton, J. I998. Contemporary hospice care: the sequestration of the unbounded body and 'dirty dying'. Sociology of Health and Illness, 2o, 2, I 2 I-43.

Lawton, J. 2000. The Dying Process. Patients' Experiences of Palliative Care. Routledge, London.

McCue, J. D. i 995. The naturalness of dying. Fournal of the American Medical Association, 273, I3, I039-45.

Mellor, P. A. I 993. Death in high modernity. In Clark, D. (ed), The Sociology of Death. Theory, Culture and Practice. Blackwell, Oxford, I I-30.

Mellor, P. A. and Shilling, C. I 993. Modernity, self-identity and the sequestration of death. Sociology, 27, 3, 4I I-3 I.

Miskella, C. and Avis, M. I998. Care of the dying person in the nursing home: exploring the care assistants' contribution. European Fournal of Oncology Nursing, 2, $80-6$.

Mitteness, L. S. and Barker, J. C. I 995. Stigmatizing a 'normal' condition: urinary incontinence in late life. Medical Anthropology Quarterly, 9, 2, г88-2 Io.

Mogielnicki, R. P., Nelson, W. A. and Dulac, J. I990. A study of the dying process in elderly hospitalised males. Fournal of Cancer Education, 5, 2, I $35-45$.

Mulkay, M. and Ernst, J. i99 I. The changing profile of social death. Archives européennes de sociologie, XXXII, I $72-96$.

Pattison, E. M. I977. The Experience of Dying. Prentice Hall, Englewood Cliffs, NJ.

Peace, S., Kellaher, L. and Willcocks, D. i997. Re-evaluating Residential Care. Open University Press, Buckingham.

Seale, C. I989. What happens in hospices: a review of research evidence. Social Science and Medicine, 28, 6, 55 I-9.

Seale, C. r 99 I. Death from cancer and death from other causes: the relevance of the hospice approach. Palliative Medicine, 5, I 2-I 9.

Seale, C. I 995. Heroic death. Sociology, 29, 4, 597-6 I 3 .

Severs, M. B. and Wilkins, P. S. W. I99 I. A hospital palliative care ward for elderly people. Age and Ageing, 2o, $36 \mathrm{I}-4$.

Sidell, M., Katz, J. and Komaromy, C. I997. Death and dying in residential and nursing homes for older people: examining the case for palliative care. Report for the Department of Health. The Open University, Milton Keynes.

Sudnow, D. i967. Passing on: the Social Organisation of Dying. Prentice-Hall, Englewood Cliff, NJ.

Sweeting, H. and Gilhooly, M. I99I-I992. Doctor, am I dead? A review of social death in modern societies. Omega, 24, 4, 25I-9.

Sweeting, H. and Gilhooly, M. i 997 . Dementia and the phenomenon of social death. Sociology of Health and Illness, r9, I, 93-I I 7.

Wilson, J. A., Lawson, P. M. and Smith, R. G. I987. The treatment of terminally ill geriatric patients. Palliative Medicine, I, I49-3.

Accepted 27 February 200I

Address for correspondence:

Centre for Cancer and Palliative Care Studies, The Institute of

Cancer Research, Royal Marsden Hospital, Fulham Road, London, $\mathrm{SW}_{3} 6 \mathrm{JJ}$.

email: froggatt@icr.ac.uk 